\title{
UML-based navigational design approach for modeling complex interactions in web applications
}

\begin{abstract}
Different complex interactions in web applications has raised new concerns on the behavior modeling approach. Web designers should designate complex interactions in web applications and provide a clear view in the design model stages. In this paper, we propose a web design model called UEWDM (UML Extensions Web Design Model) which utilizes Unified Modeling Language (UML) Profiles as the graphical notation in the design stage. UEWDM manage navigational design by two sub design models called Navigation Space Model and Navigation Access Model. Our contributions based on new UML specific modeling elements in order to support those complex interactions modeling. To validate our propose design modeling, a case study called Online Flight Booking System is provided.
\end{abstract}

Keyword: Complex interactions; Model-driven; Navigational design model; UML Profiles; Web applications 